\title{
Fine needle aspiration cytology diagnosis of Aspergilloma - A case report.
}

\author{
Dilasma Ghartimagar ${ }^{1}$, Manish Shrestha ${ }^{2}$, Arnab Ghosh $^{3}$, and Dipesh Upreti ${ }^{1}$ \\ ${ }^{1}$ Manipal College of Medical Sciences \\ ${ }^{2}$ Pokhara Academy of Health Sciences \\ ${ }^{3}$ Manipal Tata Medical College
}

March 5, 2022

\begin{abstract}
Fine needle aspiration cytology, a simple and inexpensive technique can aid in early diagnosis of aspergilloma. Here, we present a case of 55 years old female with a past history of tuberculosis. Fine needle aspiration cytology of the right lung cavitary lesion was performed which confirmed the diagnosis of aspergilloma.
\end{abstract}

\section{Introduction}

Aspergillus is a ubiquitous soil-dwelling organism that is found in humid areas, damp soil, agricultural environments, organic decay or decomposing matter. ${ }^{1}$ The risks of exposure vary both temporally and geographically and are dependent on precipitation patterns like air flow, humidity and temperature. ${ }^{2}$ Aspergillus can cause a variety of clinical syndromes ranging from mild, transient asthma to serious, disseminated disease, particularly in an immune-suppressed host. ${ }^{1}$

\section{Case Discussion}

A 55 years old female patient came with a chief complaint of cough and gradual weight loss for about 2 months. She also complained of difficulty in breathing and hemoptysis occasionally.

Laboratory investigations showed low hemoglobin $10.7 \mathrm{gm} \%$, total leukocyte count of $8,600 / \mathrm{cu}$ mm with a differential count of neutrophil $57 \%$, lymphocyte $30 \%$, eosinophils $11 \%$ and monocyte $02 \%$. Platelets were within normal limit. Her HIV, HbsAg and anti HCV tests were non reactive.

Contrast enhanced computed tomography (CECT) of lung demonstrated well defined round to oval hypodense lesion with air pockets in right upper lobe measuring approximately $38.8 \times 27.9 \times 35.1 \mathrm{~mm}$. Cavitary lesion with surrounding consolidation in right lower lobe (Fig 1) was present. Patchy consolidations with some lesions showing cavitations in left upper lobe of lung. Surrrounding reticulo-nodular opacities were also recognized (Fig 2). Multiple enlarged mediastinal nodes were also notable. Radiological impression was suggestive of pulmonary Koch's with lung abscess. Patient was referred for drainage of abscess. Under all aseptic precautions, local anesthesia and CT guidance 18 gauze spinal needle was inserted (Fig 3). Significant purulent material could not be withdrawn on aspiration as suspected on CECT. Hence aspiration was performed and slides were prepared for cytology.

Fine needle aspiration cytology showed mainly necrotic material with several clusters and balls of aspergillus (Fig 4) which demonstrated septate, acute angle branched hyphae mimicking an arborizing tree (Fig 5). Ziehl Neelsen stain for acid fast bacilli was performed which was negative. Cytological impression was given as aspergilloma of right lung. 


\section{Discussion}

Micheli in 1729 described a fungus as Aspergillus (rough head) because of the microscopic appearance of the spore-bearing structure. The first recognized infection in man due to the fungus of this genus was described by Sluyter in $1847 . .^{3}$ There are approximately 300 species of the genus aspergillus. They are within the environment, but only approximately 8 species are responsible for the vast majority of human disease. Aspergillus fumigatus is the most common pathogen accounting for most of the infections followed by aspergillus niger. ${ }^{4}$

Aspergillus species can cause various forms of lung diseases like allergic bronchopulmonary aspergillosis which is a hypersensitivity reaction to aspergillus antigens mostly due to aspergillus fumigatus. The incidence of allergic bronchopulmonary aspergillosis varies from $6 \%$ to $20 \%$ of all patients with asthma. ${ }^{5}$ Most patients are under the age of 35 years at the time of diagnosis. ${ }^{1}$ Invasive pulmonary aspergillosis is another form of pulmonary disease which is characterized by proliferation of fungal mycelia in the pulmonary parenchyma. This disease is uncommon and is due to tissue invasion with the fungi. ${ }^{1}$ Recently it has increased due to growing numbers of patients with impaired immune status associated with malignancy, organ transplantation and autoimmune conditions. ${ }^{6}$ Factors that predispose to the development of invasive aspergillosis include neutropenia, prolonged and high-dose corticosteroid therapy, advanced AIDS and chronic granulomatous disease. ${ }^{1}$ Chronic necrotizing pulmonary aspergillosis is caused by aspergillus species which is associated with cavitary infiltrates in chronic lung disease or in mild immunodeficiency state. ${ }^{6.7}$ This infection cause progressive damage to the lung parenchyma without clear evidence of tissue invasion. The patients are usually middle-aged with evidence of generalized immunosuppression in the form of diabetes mellitus, malnutrition, corticosteroid or radiation therapy. ${ }^{1}$ When aspergillus colonizes in a pre-existing lung cavity, a fungus ball comprises of fungal hyphae, inflammatory cells, fibrin, mucus and tissue debris to form an aspergilloma. ${ }^{1}$ Pulmonary aspergilloma develops in pre-existing lung cavities most commonly in patients with prior pulmonary tuberculosis but also in patients with other conditions as sarcoidosis and idiopathic pulmonary fibrosis. ${ }^{8}$ In the present case also, the patient had cavitary lesions due to past history of pulmonary tuberculosis.

Cysts and cavities are commonly encountered lesions in the lung on chest radiography and computed tomography $(\mathrm{CT})$. Solid contents within a cavity may be seen in infectious processes, such as aspergillosis and in necrotic cancer. CT can show the size, shape and precise position of cysts and cavities when these details are not apparent on chest radiography. ${ }^{7}$

The hyphae of aspergillus species range in diameter from 2.5 to $4.5 \mu \mathrm{m}$ and exhibit frequent septation. Aspergillus hyphae tend to branch dichotomously, progressively and primarily at acute angles of approximately $45^{\circ}$, mimicking an arborizing tree branch. Some times in the areas of mycelial growth, hyphae often become tangled, bulbous and distorted which may cause difficulty in identification and diagnosis. ${ }^{4}$

Fine needle aspiration cytology can be quite helpful in distinguishing malignancy from infection. ${ }^{9}$ This technique helps in detecting a wide variety of opportunistic pulmonary infections in immunocompromised patients. ${ }^{10}$ Fungal elements are detected using routine and special stains like Periodic Acid-Schiff. ${ }^{9}$ This procedure is safe, cost-effective and provides rapid results. ${ }^{10}$

Treatment of aspergilloma is considered when patients become symptomatic, usually with hemoptysis. Surgical resection is curative but may not be possible in patients with limited pulmonary function. Oral itraconazole may provide partial or complete resolution of aspergillomas in $60 \%$ of patients. Successful intracavitary treatment using CT guided percutaneous catheter instillation of amphotericin has been reported in small numbers of patients. ${ }^{8}$

\section{Conclusion}

Fine needle aspiration cytology is a simple, inexpensive and well established diagnostic technique which aid in early diagnosis and appropriate management of the patients with aspergilloma. This procedure also helps to rule out other differential diagnosis like neoplasms and inflammatory conditions.

\section{References:}


1. Amchentsev A, Kurugundla N, Saleh AG. Aspergillus-related lung disease. Respiratory Medicine CME. $2008 ; 1(3): 205-15$.

2. Patterson TF, Thompson III GR, Denning DW, Fishman JA, Hadley S, Herbrecht R, et al. Practice guidelines for the diagnosis and management of aspergillosis: 2016 update by the Infectious Diseases Society of America. Clin. Infect. Dis. 2016;63(4):e1-60.

3. Young RC, Bennett JE, Vogel CL, Carbone PP, Devita VT. The spectrum of the disease in 98 patients. Medicine. 1970;49(2):147-73.

4. Kradin RL, Mark EJ. The pathology of pulmonary disorders due to Aspergillus spp. Arch Pathol Lab Med. 2008;132(4):606-14.

5. Slavin RG, Stanczyk DJ, Lonigro AJ, Broun Sr GO. Allergic bronchopulmonary aspergillosis - A north American rarity: Clinical and immunologic characteristics. The Am. J. Med. 1969;47(2):306-13.

6. Kousha M, Tadi R, Soubani AO. Pulmonary aspergillosis: a clinical review. Eur Respir Rev. 2011;20(121):156-74.

7. Ryu JH, Swensen SJ. Cystic and cavitary lung diseases: Focal and diffuse. In: Mayo Clinic Proceedings. Elsevier; 2003. p. 744-52.

8. Giron JM, Poey CG, Fajadet PP, Balagner GB, Assoun JA, Richardi GR, et al. Inoperable pulmonary aspergilloma: Percutaneous CT-guided injection with glycerin and amphotericin B paste in 15 cases. Radiology. 1993;188(3):825-7.

9. Li W, Shetty AK, Salih ZT. Aspergillus fumigatus in a fine needle aspiration of a cavitary lung lesion. Clin. Case Rep. 2018;6(12):2475.

10. Sharma S, Gupta P, Gupta N, Lal A, Behera D, Rajwanshi A. Pulmonary infections in immunocompromised patients: The role of image-guided fine needle aspiration cytology. Cytopathology. 2017;28(1):46-54. 

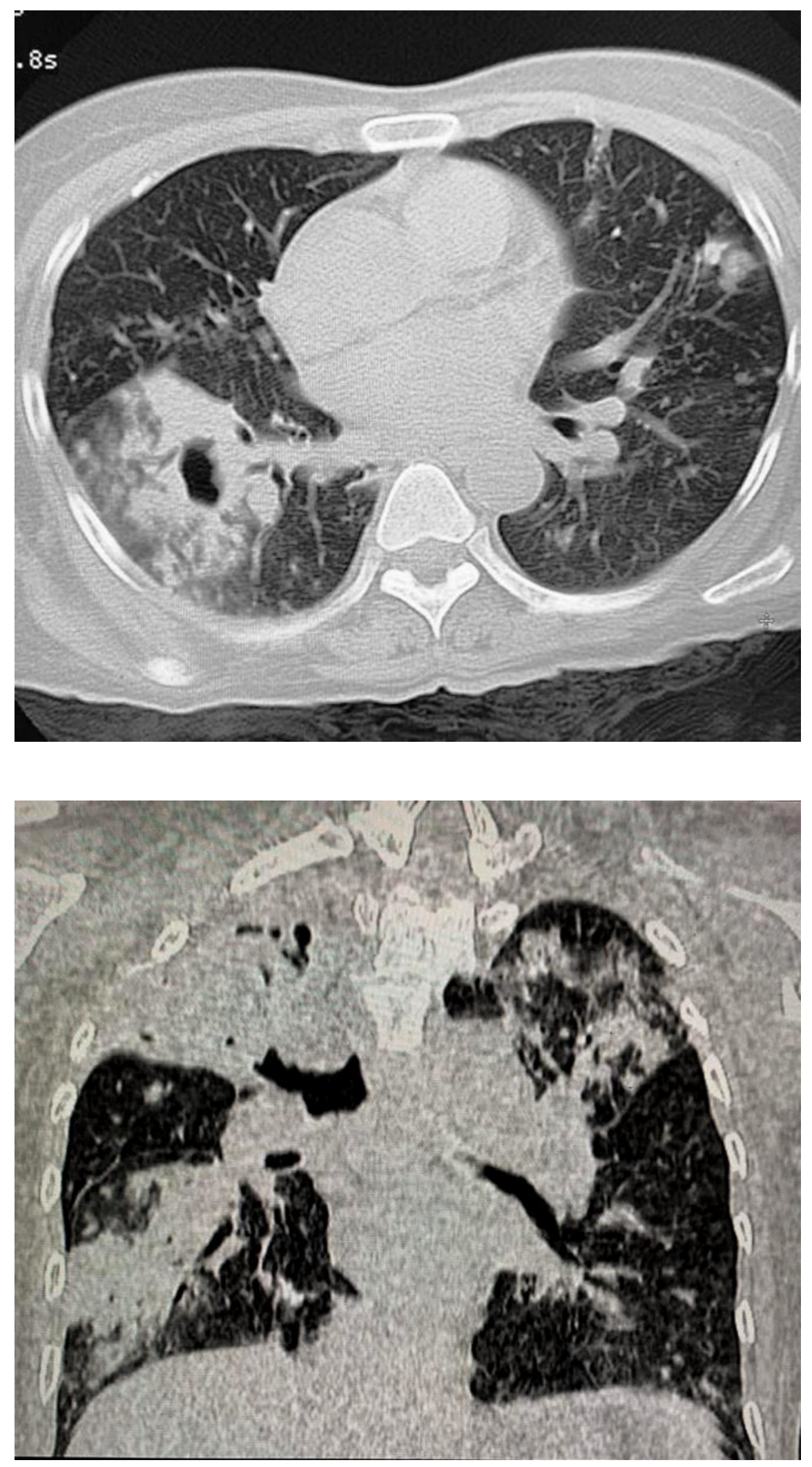

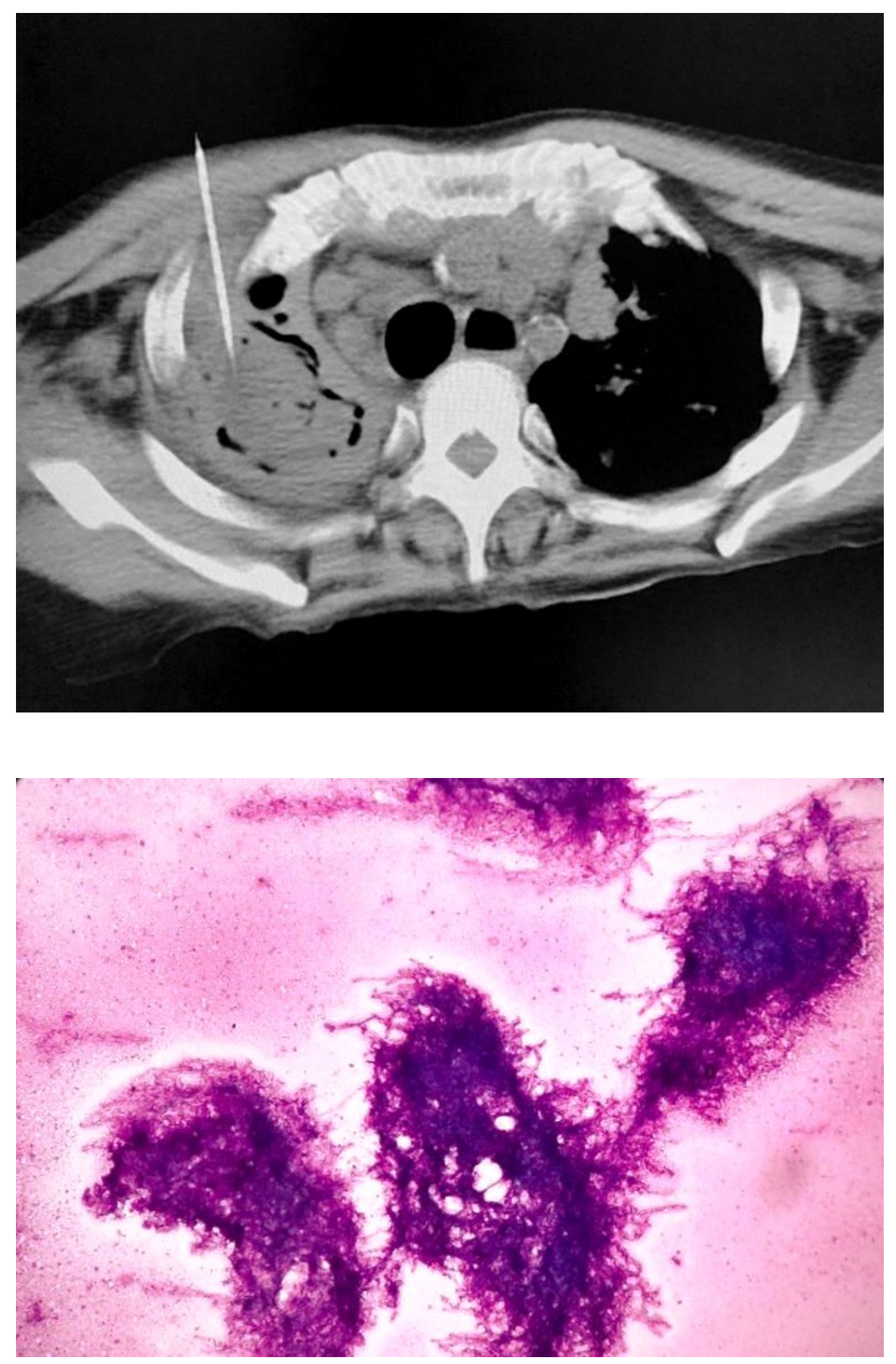


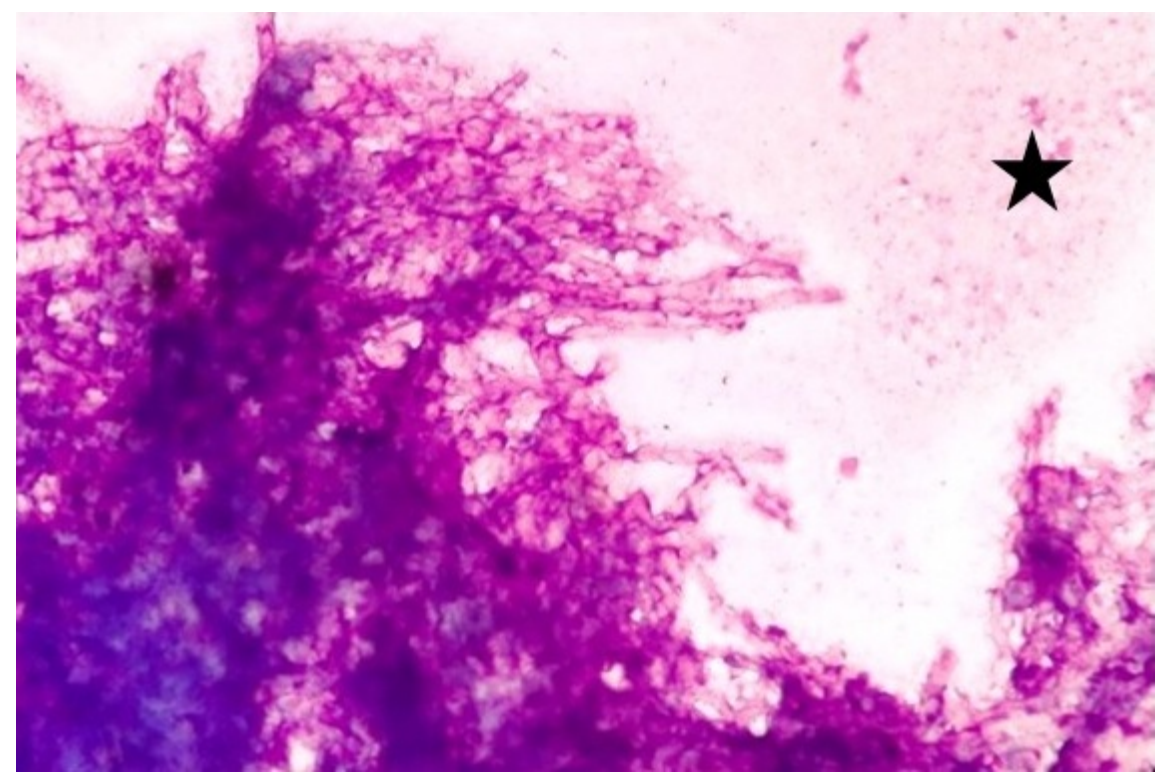

